\title{
Effect of Quality Improvement program on Health Care Workers towards Hand Hygiene at Family Practice Centers-Ismailia-Egypt
}

\author{
Reda A. Goweda ${ }^{{ }^{*}}$, Hussein M. Sharara², Mosleh A. Ismail', Abdulmageed \\ A. Abdulmageed' \\ ${ }^{1}$ Department of Family Medicine, Faculty of Medicine, Suez Canal University. ${ }^{2}$ Faculty of Commerce, Ain \\ Shams University, Egypt.
}

\begin{abstract}
Background: Hand hygiene is the most effective single measure to prevent transmission of healthcare-associated pathogens. It can be performed either by washing with soap and water or by rubbing with an alcohol-based hand rub. Aim: to identify barriers of poor adherence of Health Care Workers (HCWs) to standard hand hygiene, and to assess the impact of quality improvement program regarding hand hygiene. Subjects and Methods: This quasi-experimental study was conducted on 57 HCWs working in three Family Practice Centers in Ismailia Governorate; [physicians $(n=20)$, nurses $(n=24)$, Laboratory technicians $(n=6)$, and cleaning workers $(n=7)]$. We used the Six Sigma model as a tool for quality improvement in health care facilities. The quality improvement program towards hand hygiene was conducted over a period of 8 weeks. The impact of the intervention was assessed before and one year after the intervention using self-assessment questionnaire and observation checklist. Results: defective training and lack of scientific information were responsible for $80 \%$ of the problem (vital few) as evident from Pareto chart. About eighty-four percent (84.2\%) of the studied population achieved appropriate self-assessment after one year from intervention compared to $33.3 \%$ before the intervention $(P<0.05)$. About eighty-one percent (80.7\%) of the studied population achieved appropriate practices one year post-intervention compared to $1.80 \%$ pre-intervention $(\mathrm{P}<0.05)$. Conclusion: continuous quality improvement program could improve knowledge and practices of HCWs toward hand hygiene. Overcoming barriers as lack of training and scientific evidences are important. Implementing such program in all primary health facilities is recommended to achieve a more favorable performance.
\end{abstract}

Key words: Hand Hygiene, Infection Control, Quality Improvement.

\section{Introduction}

Hand washing with soap and water has been considered as a measure of personal hygiene for centuries. In the mid-1800s, many studies were conducted worldwide and concluded that, Hospital-Acquired Infections (HAls) were transmitted via the hands of Health Care Workers (HCWs) ${ }^{(1)}$ The current knowledge regarding transmission of pathogens through hands and prevention of infection has greatly evolved. The first international guidelines on hand hygiene were published in 2009 and recommend a range of evidence-based actions $^{(2)}$ Improving adherence with hand hygiene requires considerable effort to ensure HCWs have access to appropriate equipments/supplies and have sufficient knowledge about the importance of hand washing( ${ }^{(3)}$. Without adequate hand hygiene, hand contamination increases and

\footnotetext{
*Corresponding Author: redagoweda@yahoo.com
} 
contaminated HCWs' hands have been associated with endemic HAls. Therefore, hand hygiene is the primary measure to prevent HAls and helps in decreasing antimicrobial resistance ultimately. However, many determinants, such as lack of time, lack of equipment/supplies, and behavioral factors, often result in neglecting hand hygiene from HCWs. Although many HCWs perceive their performance as high, their adherence to hand hygiene is usually less than $40 \%$ in the absence of any interventions in this regard ${ }^{(4)}$

Hand rubs containing $60-80 \%$ alcohol are satisfactory, provided that they meet recommended standards ${ }^{(5)}$. Indeed, HCWs tend to comply more frequently with indications that protect themselves (e.g., after exposure to body fluids, after glove use, after contact with the patient or the patient's environment) ${ }^{(6)}$. The "My five moments for hand hygiene" approach merges the hand hygiene indications recommended by the WHO Guidelines into five moments when hand hygiene is required. These are: 1) Before touching a patient, 2) before clean/aseptic procedures, 3) after body fluid exposure/risk, 4) after touching a patient, and 5) after touching patient surroundings. This approach proposes a unified vision for HCWs, trainers, and observers to minimize inter-individual variation ${ }^{(7)}$

The WHO Multimodal Hand Hygiene Improvement Strategy and the WHO Implementation Toolkit have been developed to assist health care facilities to implement improvements in hand hygiene in accordance with the WHO Guidelines on Hand Hygiene in Health Care ${ }^{(8)}$ They have been pilot tested by the WHO in settings with different levels of resources and in a multicultural environment. The results were encouraging regarding knowledge and practices of HCWs. Furthermore, a substantial improvement was achieved in the facilities and equipment available for hand hygiene, including the low-cost provision of alcohol based hand rubs through local production of the WHO-recommended formulations where these were not available commercially ${ }^{(9,10)}$.

Many systems and processes guide quality improvement efforts today. These quality improvement approaches are derivatives and models of the ideas and theories developed by thought leaders as Plan-DoCheck-Act/Plan-Do-Study-Act(PDCA/PDSA), Associates for Process Improvement's (API) Model for Improvement, ISO 9000 and Six Sigma. (Six Sigma is a set of tools and strategies for process improvement. Processes that operate with "six sigma quality" over the short term are assumed to produce long-term defect levels below 3.4 defects per million opportunities ${ }^{(11)}$ )

\section{Subjects and Methods}

This is a quasi-experimental study (onegroup pretest-posttest design). The study was conducted on health care providers working in Family Practice Centers (FPCs) affiliated to Faculty of Medicine-Suez Canal University in Ismailia Governorate (EI Mahsama FPC, Abu Khalifa FPC, and Fanara FPC). The sample was a comprehensive one where all HCWs in three family practice centers were included in the study. They are categorized as physicians $(n=20)$, nurses $(n=24)$, Lab. technicians $(n=6)$ and cleaning workers $(n=7)$ with total number of 57 participants in the study.

The researcher used the Six Sigma model as a tool for quality improvement in health care facilities. The researchers passed through a process of Define, Measure, Analyze, Improve and Control (DMAIC) in this model. A Pilot study was conducted on six HCWs to test feasibility and reliability of the questions. The total number of the studied population was $51 \mathrm{HCWs}$ and completed the study to the end. The quality improvement program towards hand hygiene was conducted over a period 8 
weeks. Workshops and on job training were used in the program.

The study was conducted using selfassessment questionnaires and observational checklists as study tools. Selfassessment questionnaires included one English questionnaire designed for physicians and three Arabic questionnaires for nurses, laboratory technicians and cleaning workers. Observational checklists included the same contents of the self-assessment questionnaires. They were designed for direct observation by the researcher. One checklist was designed for observing adherence of health care providers to hand hygiene. Another one for observing structure and process related to hand hygiene in the centers. Both questionnaires and checklists were developed using the na- tional guidelines for infection control in Egypt and British guidelines for monitoring infection control within the community setting $^{(12,13)}$

The impact of this intervention was assessed by comparing the performance before and one year after the intervention using the same tools (self-assessment questionnaire and observation checklist). Informed consent was obtained from participants after sharing them the aim of the study and assuring confidentiality. Data had been coded, and fed into the computer. The statistical package for social sciences (SPSS version 20.0) was used for data management. The data were presented in tables and graphs. Chi-square test was used to test the statistical significance of categorical data.

Table 1: Distribution of the studied population according to their job description

\begin{tabular}{|l|c|}
\hline Health Care Workers $(\mathrm{n}=51)$ & No $(\%)$ \\
\hline Physicians & $20(35 \%)$ \\
Nurses & $24(42 \%)$ \\
Laboratory Technicians & $6(11 \%)$ \\
Cleaning Workers & $7(12 \%)$ \\
\hline
\end{tabular}

\section{Results}

The current study was conducted on all health care Workers (HCWs) working in Family Practices Centers affiliated to Faculty of Medicine, Suez Canal University (FOM/SCU). HCWs included physicians $(n=20) 35 \%$, nurses $(n=24) 42 \%$, laboratory technicians $(n=6) 11 \%$ and cleaning workers $(n=7) 12 \%$ with total number of 51 participants in the study as shown in table (1). Female predominance was observed among physicians ( $85 \%)$, nurses $(87 \%)$, and technicians (100\%). Ninety-five percent of physicians, $62.4 \%$ of nurses, and $83.4 \%$ of laboratory technicians were in the age group of $\geq 25$ years. Sixty percent of the studied physicians have practical experi- ences for $\geq 5$ years comparable with $79.2 \%$ for nurses and $66.6 \%$ for laboratory technicians. No HCWs received any annual checkup. Only $25 \%$ of the studied physicians, $12.5 \%$ of nurses, none of laboratory technicians and cleaning workers received Hepatitis $B$ Vaccine (HBV). Regarding workload of the studied physicians, $45 \%$ of them consulted up to 10 patients per days in daily practice, similar percent $(40 \%)$ consulted patients at a rate of $10-20$ daily. Only $15 \%$ of them had consulted more than 20 patients per day. Pareto chart was used to identify the vital few causes of poor adherence to hand hygiene. (Pareto chart is a type of chart that contains both bar and line graph where individual values are represented in descending order by bars and the cumulative total is represented by the line). It is evident that, defective training and lack of 
scientific information were responsible for $80 \%$ of causes of poor adherence to hand hygiene vital few) while the other causes account for $20 \%$ of the problem (useful many) as addressed in Pareto chart (Figure 1). Remedy no (3) was the most effective remedy to be implemented according to the set of proposed criteria (total score was 19). This remedy (improvement intervention) included training courses for CHWs towards hand hygiene by researcher in the centre (on job training along with interactive lectures) as addressed in table (2).

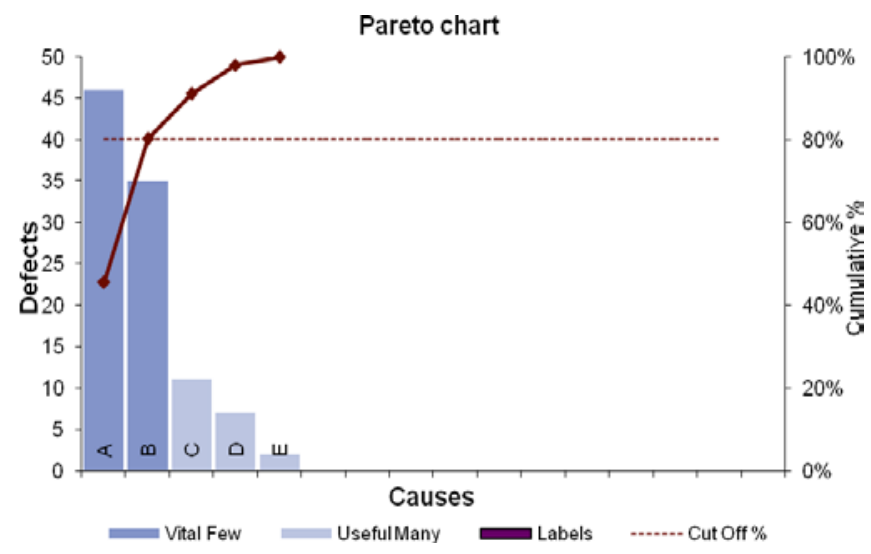

Figure 2: Causes of poor adherence to standard hand hygiene (Pareto chart). A: Defected training, B: Lack of scientific information: Increase rate of patients; D: Lack of resources: lack of super vision and monitoring

Concerning self-assessment of the studied population, there was a statistically significant difference between pre and post intervention [one year] $(\mathrm{P}<0.05)$ regarding the hand hygiene as shown in table (3). About eighty four percent (84.2\%) of the studied population achieved appropriate self-assessment (>80\%) after one year from intervention comparable with $33.3 \%$ before the intervention. It is evident that physicians, nurses and cleaning workers showed significant statistical difference before and after the intervention regarding selfassessment of hand hygiene $(P<0.05)$ as demonstrated in table (4).

Table 2: Remedies for improvement of defective training regarding hand hygiene

\begin{tabular}{|l|ccc|}
\hline Criteria & Remedy 1 & Remedy 2 & Remedy 3 \\
\hline 1- Cost (least cost take score 3) & 1 & 3 & 3 \\
2- Impact on problem & 1 & 2 & 3 \\
3- Benefit from remedy & 1 & 3 & 3 \\
4-Implementation time & 2 & 2 & 3 \\
5-Resistance to change & 1 & 1 & 2 \\
6-Certainty about effectiveness & 1 & 2 & 2 \\
7-Health and environment & 3 & 2 & 3 \\
Total scores & 10 & 15 & 19 \\
\hline
\end{tabular}

Remedy 1: Training courses for CHWs towards hand hygiene by team from MOHP; Remedy 2: Training courses for CHWs towards hand hygiene by Infection control (IC) team of Suez Canal University Hospital affiliated to SCU; Remedy 3: Training courses for $\mathrm{CHWs}$ towards hand hygiene by researcher in the centre (on job training along with interactive lectures). 
Table 3: Comparison of Pre-post intervention regarding self-assessment of studied population towards hand hygiene

\begin{tabular}{|l|ccr|}
\hline Self-assessment regard- & Pre intervention & Post-intervention (1 year) & P value \\
ing hand hygiene & No. $(\%)$ & No. $(\%)$ & \\
\hline Appropriate $(>80 \%)$ & $19(33.3)$ & $48(84.2)$ & \\
Inappropriate $(<80 \%)$ & $38(66.7)$ & $9(15.8)$ & $0.000^{*}$ \\
\hline
\end{tabular}

* Statistically significant difference ( $\mathrm{p}$-value $<0.05)$; Total No $=57$

Table 4: Comparison of pre and post intervention self-assessment of the studied population regarding hand hygiene

\begin{tabular}{|l|l|ccc|}
\hline $\begin{array}{l}\text { Groups of studied } \\
\text { population }\end{array}$ & $\begin{array}{l}\text { Hand hygiene } \\
\text { (self-assessment) }\end{array}$ & $\begin{array}{c}\text { Pre- intervention } \\
\text { No. }(\%)\end{array}$ & $\begin{array}{c}\text { Post-intervention (1 year) } \\
\text { No. (\%) }\end{array}$ & P value \\
\hline Physicians $(\mathrm{n}=20)$ & Appropriate & $9(45)$ & $19(95)$ & \\
& Inappropriate & $11(55)$ & $1(5)$ & $0.001^{*}$ \\
\hline Nurses $(\mathrm{n}=24)$ & Appropriate & $8(33.2)$ & $20(83.3)$ & \\
& Inappropriate & $16(65.8)$ & $4(16.7)$ & $0.001^{*}$ \\
\hline Technicians $(\mathrm{n}=6)$ & Appropriate & $2(33.3)$ & $5(83.3)$ & \\
& Inappropriate & $4(66.6)$ & $1(16.7)$ & 0.121 \\
\hline Workers $(\mathrm{n}=7)$ & Appropriate & 0 & $4(57.1)$ & \\
& Inappropriate & $7(100)$ & $3(42.9)$ & $0.021^{*}$ \\
\hline
\end{tabular}

* Statistically significant difference ( $p$-value $<0.05)$

Table (5) shows there was highly significant difference between pre and post intervention (one year) regarding direct observation of hand Hygiene practice of the studied population. About eighty one percent (80.7\%) of the studied population achieved appropriate practices $(>80 \%)$ in post intervention compared to $1.80 \%$ before inter- vention. All HCWs (physicians, nurses, laboratory technicians and cleaning workers) demonstrated significant improvement in practices related to hand hygiene one year after the intervention comparable with before intervention $(p<0.05)$ as shown in table (6).

Table 5: Practices of studied population regarding hand hygiene by direct observation (Pre-post intervention)

\begin{tabular}{|l|cc|c|}
\hline $\begin{array}{l}\text { Direct observation regarding hand } \\
\text { hygiene }\end{array}$ & $\begin{array}{c}\text { Pre- intervention } \\
\text { No. }(\%)\end{array}$ & $\begin{array}{c}\text { Post-intervention (1 year) } \\
\text { No. }(\%)\end{array}$ & P-value \\
\hline Appropriate $(>80 \%)$ & $1(1.8)$ & $46(80.7)$ & \\
Inappropriate $(<80 \%)$ & $56(98.2)$ & $11(19.3)$ & $0.000 *$ \\
\hline
\end{tabular}

* Statistically significant difference ( $p$-value $<0.05$ ); Total No. $=57$

\section{Discussion}

The current study was carried-out on 57 HCWs working at family practice centers affiliated to FOM/SCU -Ismailia Governorate. It aimed at identifying barriers of poor adherence of HCWs to standard hand hygiene. In addition, it assessed the impact of quality improvement program regarding hand hygiene. On studying barriers (causes) of non-adherence among HCWs, it was found that most of the studied population 
(45.6\%) think that the non-adherence due to lack of training. Lack of scientific information as a barrier to adherence to standard hand hygiene was mentioned by $35 \%$ of the studied population. Other barriers were addressed as consulting a high rate of patient daily $10.6 \%$ and lack of required resource $(7.1 \%)$. These findings were in partial agreement with other studies reported that lack of awareness of the risk of getting infection and lack of knowledge of guidelines related to hand hygiene were perceived as barriers to good hand hygiene practices. Furthermore, some HCWs believed that they washed their hands when necessary even when observations indicated that they did not ${ }^{(14)}$

Table 6: Practices of different groups of studied population regarding hand hygiene by direct observation (Pre-post intervention)

\begin{tabular}{|l|l|ccc|}
\hline $\begin{array}{c}\text { Groups of studied } \\
\text { population }\end{array}$ & $\begin{array}{c}\text { Hand hygiene } \\
\text { (direct observation) }\end{array}$ & $\begin{array}{c}\text { Pre- intervention } \\
\text { No. (\%)) }\end{array}$ & $\begin{array}{c}\text { Post-intervention (1 year) } \\
\text { No. (\%) }\end{array}$ & P value \\
\hline Physicians ( $\mathrm{n}=20)$ & Appropriate & $1(5)$ & $17(85)$ & \\
& Inappropriate & $19(95)$ & $3(15)$ & $0.000^{*}$ \\
\hline Nurses $(\mathrm{n}=24)$ & Appropriate & 0 & $2083.3)$ & \\
& Inappropriate & $24(100)$ & $4(16.7)$ & $0.000^{*}$ \\
\hline Technicians $(\mathrm{n}=6)$ & Appropriate & 0 & $4(66.7)$ & \\
& Inappropriate & $6(100)$ & $2(33.3)$ & $0.000^{*}$ \\
\hline Workers $(\mathrm{n}=7)$ & Appropriate & 0 & $5(71.4)$ & \\
& Inappropriate & $7(100)$ & $2(28.6)$ & $0.000^{*}$ \\
\hline
\end{tabular}

* Statistically significant at $p<0.05$

Concerning self-assessment of the studied population, there was a statistically significant difference between pre and post intervention [one year] $(P<0.05)$ regarding hand hygiene. About eighty four percent $(84.2 \%)$ of the studied population achieved appropriate self-assessment $(>80 \%)$ after one year from intervention comparable with $33.3 \%$ before the intervention. It is evident that physicians, nurses and cleaning workers showed significant statistical difference before and after the intervention regarding self-assessment of hand hygiene $(P<0.05)$. The results of the current study were consistent with the reported results from numerous studies. Our study showed that educational programs can effectively increase knowledge, and positive attitudes which ensures adherence with international protocols, and regulations for the prevention and control of infection ${ }^{(15)}$. Such concordance might be explained in the view of adherence of HCWs worldwide to a new behavior. This could be fostered maintained as shown after one year in the current study by overcoming barriers to such behavior particularly training and updated knowledge regardless their job. The direct observation of practices of CHWs is more objective than self-assessment because it reflects the reality. It was evident that, there was highly significant difference between the pre and the post intervention (one year) regarding direct observation of adherence to practices of good hand hygiene among the studied population. About eighty one percent $(80.7 \%)$ of the studied population achieved appropriate practice $(>80 \%)$ in the post intervention compared to $1.80 \%$ in the pre intervention. All health care providers (physicians, nurses, laboratory technicians and cleaning workers) demonstrated significant improvement in practices related to hand hygiene one year after the intervention comparable with before intervention $(p<0.05)$. 
These results were not in full agreement with the reported results from European society. In the final report of the Clean Hands Save Lives Campaign which Was conducted in New South Wales between November 2005-May 2007, it was found that overall hand hygiene adherence between February 2006 and February 2007 improved from $47.1 \%$ to $62.2 \%{ }^{(16)}$. Also, adherence of HCWs to recommended hand hygiene procedures has been unacceptably poor, with mean baseline rates ranging from $5 \%$ to $81 \%$, with an overall average of about $40 \%{ }^{(17)}$. In an epidemiological study of HH carried out in 1994 in hospitals affiliated to the University of Geneva, an average rate of compliance of $48 \%$ was observed $^{(18)}$. Generally there is poor adherence with hand hygiene regulations by health care workers all over the world, and all the studies carried out in hospitals suggest that the frequency of adherence is lower than $50 \%{ }^{(18)}$. The relative limited number of the studied population could explain such discrepancy between the results of the current study, and the abovementioned one, and the researcher being in touch with them frequently makes them more adherent to the new behavior. On the other hand, most of the reported results from hospital based studies not primary care. In addition, they were more motivated to be accredited from the national body belonging to MOHP at the time of conducting the study.

\section{Conclusions}

Continuous quality improvement intervention could improve knowledge and practices of health care workers toward hand hygiene. This will be achieved after overcoming the barriers such as lack of training, and scientific information that were evident in the current study. Implementing such pro- gram in all primary health care facilities is recommended.

\section{References}

1. World Health Organization (WHO) Guidelines on Hand Hygiene in Health Care. Geneva. (2009) http://whqlibdoc. who.int/publications/2009/9789241597906 eng. pdf. Accessed July 20, 2011.

2. Centers for Disease Control and Prevention (CDC): Hand Hygiene in Healthcare Settings.http://www.cdc.gov/HandHygiene /index.html [Accessed July 20, 2011].

3. Tai JW, Mok ES, Ching PT, Seto WH, Pittet D. Nurses and physicians' perceptions of the importance and impact of healthcareassociated infections and hand hygiene: a multi-center exploratory study in Hong Kong. Infection.2009; 37:320-33.

4. Erasmus V, Daha TJ, Brug H, Richardus JH, Behrendt MD, Vos MC. Systematic review of studies on compliance with hand hygiene guidelines in hospital care. Infect Control Hosp Epidemiol 2010; 31:283- 94.

5. Rott er M, Satt ar S, Dharan S, Allegranzi B, Mathai E, Pittet D. Methods to evaluate the microbicidal activities of hand-rub and hand-wash agents. J Hosp Infect 2009; 73:191-9.

6. Borg MA, Benbachir M, Cookson BD, Redjeb SB, Elnasser Z, Rasslan O. Selfprotection as a driver for hand hygiene among healthcare workers. Infect Control Hosp Epidemiol 2009; 30:578-80.

7. Sax H, Allegranzi B, Uçkay I, Larson E, Boyce J, Pittet D. "My five moments for hand hygiene": a user-centred design approach to understand, train, monitor and report hand hygiene. J Hosp Infect 2007; 67:9-21.

8. WHO. Implementation Toolkit (2010). Available from: http://www.who.int/ gpsc/ 5may/tools/en/index.html [Accessed July 20, 2011].

9. WHO: Guide to local production (2010): WHO recommended hand rub formulations. Available from: http://www.who. int/gpsc/5may/ Guide to Local Production.pdf [Accessed July 20, 2011]. 
10. Kampf G, Marschall S, Eggerstedt S, Ostermeyer C. Efficacy of ethanol-based hand foams using clinically relevant amounts: a crossover controlled study among healthy volunteers. BMC Infect Dis 2010; $10: 78$.

11. Stoeck M. Quality improvement systems, theories and tools. In: Ransom SB, Joshi MS, Nash DB. (ed): The Health care Quality Book. Vision, Strategy and Tools.2005: 6386. Health Administration Press, Chicago, IL, AUPHA Press, Washington, D.C.

12. Ministry of Health and Population (MOHP). The national guideline for infection control, Egypt. (2004): Available from: www.drguide.mohp.gov.eg/NewSite/Elearning/InfectionControl/infctrlp1.asp).

13. Infection Control Nurses Association; Audit tools for monitoring infection control guidelines within the community setting 2005: Department of Health, England. Available from: www.icna.co.uk
14. Dubbert PM, Dolce J, Richter W, Miller M, Chapman SW. Increasing ICU staff hand washing: effects of education and group feedback. Infect Control Hospi Epidemiol 1990; 11:191-193

15. Huang J, Jiang D, Wang X, Liu Y, Fennie K, Burgess J, Williams AB. Changing knowledge, behaviour, and practice related to universal precautions among hospital nurses in China. Journal of Continuing Education in Nursing.2002; 23(5):217-224.

16. Pantle $A$ and Fitzpatrick K. The final report of the Clean Hands Save Lives Campaign New South Wales Nov. 2005- May 2007.

17. Pittet D. Hand Hygiene and patient care: pursuing the Semmelweis legacy. Lancet Infect Dis 2001; 9-20.

18. Pittet D, Hugonnet $S$, Harbarth $S$, Mourouga P, Sauvan V, Touveneau S, Perneger TV. Effectiveness of a hospitalwide program to improve compliance with hand hygiene. Infection Control Program.Lancet 2000; 356:1307-12. 